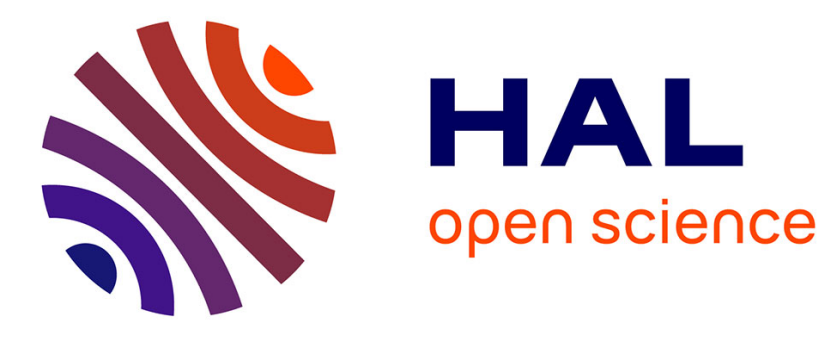

\title{
On Steady-State Cornering Analysis for Motorcycles
} Majda Fouka, Lamri Nehaoua, Hichem Arioui, Saïd Mammar

\section{To cite this version:}

Majda Fouka, Lamri Nehaoua, Hichem Arioui, Saïd Mammar. On Steady-State Cornering Analysis for Motorcycles. IEEE Intelligent Transportation Systems Conference (ITSC 2019), Oct 2019, Aukland, New Zealand. pp.3782-3787, 10.1109/ITSC.2019.8916883 . hal-02173422

\section{HAL Id: hal-02173422 \\ https://hal.science/hal-02173422}

Submitted on 4 Jul 2019

HAL is a multi-disciplinary open access archive for the deposit and dissemination of scientific research documents, whether they are published or not. The documents may come from teaching and research institutions in France or abroad, or from public or private research centers.
L'archive ouverte pluridisciplinaire HAL, est destinée au dépôt et à la diffusion de documents scientifiques de niveau recherche, publiés ou non, émanant des établissements d'enseignement et de recherche français ou étrangers, des laboratoires publics ou privés. 


\title{
On Steady-State Cornering Analysis for Motorcycles
}

\author{
Majda Fouka ${ }^{1}$, Lamri Nehaoua ${ }^{1}$, Hicham Arioui $^{1}$ and Said Mammar ${ }^{1}$
}

\begin{abstract}
In this paper, a neutral-path departure algorithm is proposed to define safe handling threshold conditions and dangerous steering situation for powered two-wheeled vehicles. Based on this study, a Self Steering Gradient for motorcycles is proposed as a risk function for neutral-path departure detection. Furthermore, the motorcycle overturning or understeering are analyzed based on the handling index. This index depends on the intrinsic motorcycle parameters, as well as, the state outputs. The proposed neutral-path departure algorithm aims to assess the risk when the motorcycle begins to drift out of the neutral path. Finally, the effectiveness of the detection scheme is tested using a high-fidelity software BikeSim ${ }^{\circledR}$.
\end{abstract}

Index Terms-Steady cornering, Risk function, Motorcycle Safety.

\section{INTRODUCTION}

Recently, car manufacturers are constantly seeking to design new intelligent safety systems, in order, to detect malfunctions in riding task and to improve the performance and reliability of active safety systems. Lane departures case account for a significant percentage of roadway fatalities. According to Federal Highway Administration's Roadway (FHWA's), from 2015 to 2017 an average of 19,23 traffic fatalities resulted from roadway departures crashes [1], [3]. In-Depth Investigations of motorcycle crashes, show that human errors due to tiredness or temporarily distraction are the most important factors. This is why the last few years have seen the emergence of on-board roadway departure assistance systems in cars as a way of improving security and helping to avoid damage or even fatal crashes in dangerous steering situations [4]-[8]. In spite of the fact that roaddeparture system is present in every modern car, it is not yet developed for motorcycle and those implemented for fourwheeled vehicles are not entirely transferable to motorcycles due to the fact that motorcycle dynamics is more complex and unstable. Therefore, departure avoidance systems for motorcycle are the next step, aimed to detect as early as possible, when the motorcycle is involuntary getting out of the lane. Then, the rider corrects his trajectory, maintain stability and keep acceptable performances by means of this early detection systems.

Currently, relevant works are intended to study the design of Lane Departure Warning for Motorcycle (LDWM) from the control point of view. The control effects of the steerby-wire (SBW) system for motorcycles was discussed in [9]. Lane-keeping controller was evaluated through computer simulation with a rider-control model in [10], [11], in which the lane-following performance was improved by using a

1 Authors are with University of Evry Val d'Essonne - Paris Saclay, IBISC Laboratory, Evry, France majda. fouka@univ-evry. fr virtual-point regulator. In [12], the authors developed a lane change decision aid system (LCDAS) under the weather and environmental change using a single camera. In [13], [14], author study the motorcycle's steering behavior, achieved by the vision-based approach to define the motorcycle dynamic position on the road and detect under or oversteer situation. Lane Departure Warning System for a motorcycle is still under development and needs a more thorough investigation to be implemented in new bikes.

A key problem in building up departure warning systems for motorcycle or even vehicles is how to develop a driving risk function, which can be used to warn the rider in the case of passive assistance or engage the control action in the case of active assistance. Car roadway departure system usually defines a lane crossing Time (TLC) and distance to lane crossing (DLC) as a risk index, to assess the time for involuntary trespassing the boundaries, see [5], [6]. Nevertheless, the TLC presents some limitations, it requires accurate road information, moreover, it is approximated geometrically without vehicle dynamics consideration to integrate driver corrections. Also the TLC alone is not adequate for imminent departure situation or not sufficient to characterize road-departure situations. Indeed, even in risk situation of high speed in longitudinal motion and/or overmuch lateral dynamic, in this case, a great value of TLC can be expected with no alarm generation, see [15].

Among other, steady-state analysis and handling capabilities issues are very related to vehicle safe trajectory and roadway departure. Many researches were devoted to study the steady-state handling for cars, see [16]-[19], either to define analytical handling criteria or the critical dynamic variables with which the divergent loss of handling occurs. The analysis of the properties of handling highlights certain dynamic aspects that are important to define dangerous/safe stability threshold conditions [20], as the neutral, overturning or underturning behavior [18]-[22]. Unfortunately, this keen interest is not as evident to some other road users. Inspired by steady-state and the handling analysis for cars, our present work tackles the question of the motorcycle's steering behavior based on the stationary cornering condition, followed by the design of a new risk indicator for motorcycle, to describe steering neutral, under or over behavior.

In this paper, a detection approach towards getting circular stationary states and analytical handling conditions is developed for powered two wheeled vehicles (PTWv). Based on the established motorcycle model, combined with magic formula tire cornering forces, a Self Steering Gradient for motorcycles " $S_{S}$ " is proposed as a risk function. The handling index " $S_{S}$ " is computed from currently available standard 
sensors: Inertial Measurement Unit (IMU), steering encoder and Global Navigation Satellite System (GNSS) without the need on state observer. Furthermore, a neutral-path departure (NPD) algorithm based on the " $S_{S}$ " is proposed to characterize the motorcycle steering behavior: over or under-steering situations. The algorithm monitors signals from sensors and compares intended neutral (theoretical) and actual paths. If the trajectories differ from each other, this means that motorcycle is going out neutral path, in this case, the algorithm generates an alarm to warn the rider.

The paper is organized as follow: Section II presents the lateral motorcycle dynamics. Section III presents the steady steering behavior and handling analysis for motorcycle. In section IV, the side slip relations are examined to define a risk function for the steering behavior. From which the proposed NPD algorithm is analyzed in section $\mathrm{V}$ and evaluated using BikeSim in section VI. Last, section VII concludes the paper.

\section{LATERAL MOTORCYCLE DYNAMICS}

In this section, the lateral motion of the motorcycle is modeled as a single track vehicle, as shown in Fig. 1. This model has three degrees of freedom, namely the lateral displacement, roll, and yaw motion, including the tire cornering properties, described by the following differential equations:

$$
\left\{\begin{aligned}
m\left(\dot{v}_{y}+\dot{\psi} v_{x}\right) & =F_{y f}+F_{y r} \\
I_{z} \ddot{\psi} & =l_{f} F_{y f}-l_{r} F_{y r} \\
I_{x} \ddot{\phi}+m h\left(\dot{v}_{y}+\dot{\psi} v_{x}\right) & =m h g \phi \\
m a_{y} & =F_{y f}+F_{y r}
\end{aligned}\right.
$$

Where $F_{y f}$ and $F_{y r}$ are the lateral forces on the front and rear wheels, $v_{x}$ is the forward speed, $\psi$ is the yaw rate, $a_{y}$ is the lateral acceleration, $m$ is the motorcycle mass, $l_{f}$ and $l_{r}$ are horizontal distances, $h$ is the height of the gravity center, and $I_{z}$ and $I_{x}$ are the moment of inertia with respect to the $z$-axis and $x$-axis respectively.

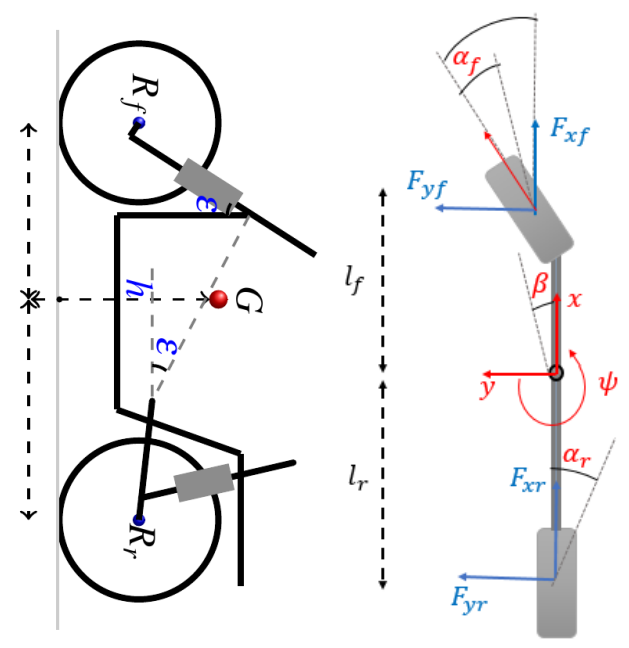

Fig. 1: Motorcycle kinematics.
The lateral cornering forces are given by:

$$
\left\{\begin{aligned}
F_{y f} & =-C_{f 1} \alpha_{f}+C_{f 2} \gamma_{f} \\
F_{y r} & =-C_{r 1} \alpha_{r}+C_{r 2} \gamma_{r}
\end{aligned}\right.
$$

where $C_{f 1}, C_{f 2}, C_{r 1}$ and $C_{r 2}$ are the cornering stiffness and camber coefficients, $\alpha_{f}$ and $\alpha_{r}$ are sideslip angles, $\gamma_{f}$ and $\gamma_{r}$ are the camber angles of the front and rear tyres, respectively. With :

$$
\left\{\begin{aligned}
\alpha_{f} & =\frac{v_{y}+l_{f} \dot{\psi}}{v_{x}}-\delta \cos (\varepsilon) \\
\gamma_{f} & =\phi+\delta \sin \varepsilon \\
\alpha_{r} & =\frac{v_{y}-l_{r} \dot{\psi}}{v_{x}} \\
\gamma_{r} & =\phi
\end{aligned}\right.
$$

After slight calculation, one can obtain:

$$
\left\{\begin{array}{l}
m\left(\dot{v}_{y}+\dot{\psi} v_{x}\right)=a_{1} \frac{v_{y}}{v_{x}}+a_{2} \frac{\dot{\psi}}{v_{x}}+a_{3} \delta+a_{4} \phi \\
I_{z} \ddot{\psi}=a_{5} \frac{v_{y}}{v_{x}}+a_{6} \frac{\dot{\psi}}{v_{x}}+a_{7} \delta+a_{8} \phi \\
I_{x} \ddot{\phi}+m h\left(v_{y}+\dot{\psi} v_{x}\right)=m h g \phi
\end{array}\right.
$$

whereas, $a_{i}$ are function of $l_{f}, l_{r}, \varepsilon, C_{f i}$ and $C_{r i}$ with $i=$ $(1,2)$, given by:

$$
\left\{\begin{array}{l}
a_{1}=-\left(C_{f 1}+C_{r 1}\right) \\
a_{2}=-\left(l_{f} C_{f 1}-l_{r} C_{r 1}\right) \\
a_{3}=\left(C_{f 1} \cos (\varepsilon)+C_{f 2} \sin (\varepsilon)\right) \\
a_{4}=\left(C_{f 2}+C_{r 2}\right) \\
a_{5}=a_{2} \\
a_{6}=-\left(l_{f}^{2} C_{f 1}+l_{r}^{2} C_{r 1}\right) \\
a_{7}=\left(l_{f} C_{f 1} \cos (\varepsilon)+l_{f} C_{f 2} \sin (\varepsilon)\right) \\
a_{8}=\left(l_{f} C_{f 2}-l_{r} C_{r 2}\right)
\end{array}\right.
$$

\section{Steady Steering Behaviour And Handling ANALYSIS}

The aim of this section is to extract form the above model (4), the operating steady steering conditions. These characteristics are important and concur to define the sensitivity of the motorcycle's handling [23]. Which is commonly judged by how a vehicle reacts to the rider inputs during cornering. Under a steady cornering scenario, the yaw rate $\dot{\psi}$ as well as the steering angle, the lateral velocity and the side slip are constants, it follows:

$$
\left\{\begin{array}{l}
m \dot{\psi} v_{x}=a_{1} \frac{v_{y}}{v_{x}}+a_{2} \frac{\dot{\psi}}{v_{x}}+a_{3} \delta+a_{4} \phi \\
a_{5} \frac{v_{y}}{v_{x}}+a_{6} \frac{\dot{\psi}}{v_{x}}+a_{7} \delta+a_{8} \phi=0 \\
\dot{\psi} v_{x}=g \phi
\end{array}\right.
$$

After quick manipulation, one can write :

$$
[\underbrace{\left(a_{5} m-\frac{a_{5} a_{4}-a_{1} a_{8}}{g}\right)}_{K_{1}} v_{x}^{2} \underbrace{-\left(a_{5} a_{2}-a_{1} a_{6}\right)}_{K_{2}}] \frac{\dot{\psi}}{v_{x}}=\underbrace{\left(a_{5} a_{3}-a_{1} a_{7}\right)}_{K_{3}} \delta
$$

where

$$
\left\{\begin{array}{l}
K_{1}=\left(-\left(l_{f} C_{f 1}-l_{r} C_{r 1}\right) m-\frac{\left(l_{f}+l_{r}\right)\left(C_{f 2} C_{r 1}-C_{f 1} C_{r 2}\right)}{g}\right) \\
K_{2}=\left(l_{f}-l_{r}\right)^{2} C_{f 1} C_{r 1} \\
K_{3}=\left(l_{f}+l_{r}\right) C_{f 1}\left(C_{r 1} \cos (\varepsilon)-C_{f 2} \sin (\varepsilon)\right)
\end{array}\right.
$$


The steering sensitivity $\frac{\dot{\psi}}{\delta}$ is given by:

$$
\frac{\dot{\psi}}{\delta}=\frac{\left(\frac{K_{3}}{K_{2}}\right) v_{x}}{\left[K v_{x}^{2}+1\right]}
$$

Where $K=\frac{K_{1}}{K_{3}}$ is the handling factor. The aim of this part is to extract from the above model (4), the operating steady steering conditions. The motorcycle steering tendency depends on the yaw rate, the forward velocity $v_{x}$ and the stability factor $K$, it follows:

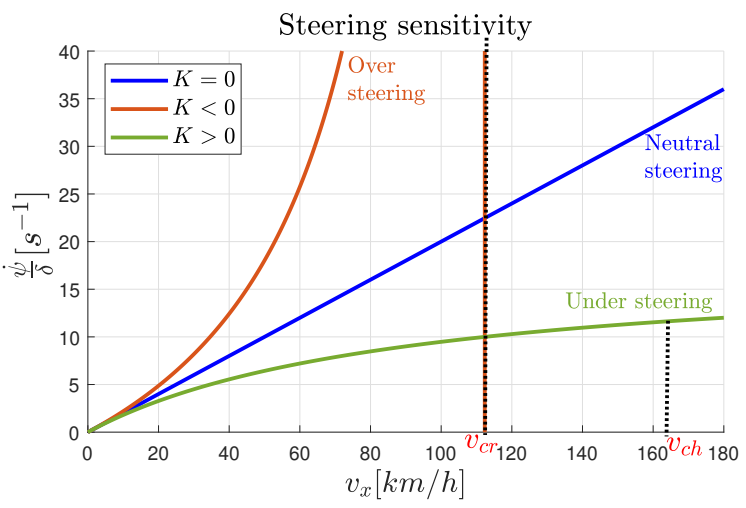

Fig. 2: Motorcycle sensitivity gain.

1) $K=0$ for Neutral steering, $\frac{\dot{\psi}}{\delta}=\frac{K_{3}}{K_{2}} v_{x}$ has a linear relation with motorcycle speed with $\frac{K_{3}}{K_{2}}$ is the slope.

2) $K>0$ Under-steering, the steering sensitivity is below the neutral steering characteristic. $\frac{d}{d v_{x}}\left(\frac{\dot{\psi}}{\delta}\right)=0 \rightarrow v_{c h}=$ $\frac{1}{\sqrt{K}}$. It is interpreted as the motorcycle characteristic speed at which the vehicle reacts most sensitively to steering inputs.

3) $K<0$ Over-steering: when $v_{c r}=\frac{1}{\sqrt{-K}}$, the steering sensitivity strives toward infinity, where $v_{c r}$ is the critical speed, for which a motorcycle becomes unstable because its steering is canceled, as even very small steering input would lead to infinite yaw rate.

\section{THE SIDE SLIP DYNAMICS}

The following study defines a new handling factors proper to motorcycle. In steady cornering, the state variables are given by:

$$
\phi=\frac{\dot{\psi} v_{x}}{g}, \quad \rho=\frac{\dot{\psi}}{v_{x}}=\frac{1}{R}, \quad a_{y}=\dot{\psi} v_{x}
$$

The side slip relation can be expressed as a function of motorcycle intrinsic and dynamic variables from equations (1 and 2): $\alpha_{f}-\alpha_{r}=f_{1}\left(\phi, \delta, a_{y}, \varepsilon, m, l_{f}, l_{r}, C_{f i}, C_{r i}\right)$, as well as from the kinematics equation (3): $\alpha_{f}-\alpha_{r}=f_{2}\left(\delta, R, \varepsilon, l_{f}, l_{r}\right)$. Now, replacing cornering forces (2) into (1), it follows:

$$
\left[\begin{array}{c}
\alpha_{f} \\
\alpha_{r}
\end{array}\right]=\left[\begin{array}{cc}
C_{f 1} & C_{r 1} \\
l_{f} C_{f 1} & -l_{r} C_{r 1}
\end{array}\right]^{-1}\left(\left[\begin{array}{c}
m a_{y} \\
0
\end{array}\right]-\left[\begin{array}{cc}
C_{f 2} & C_{r 2} \\
l_{f} C_{f 2} & -l_{r} C_{r 2}
\end{array}\right]\left[\begin{array}{c}
\gamma_{f} \\
\gamma_{r}
\end{array}\right]\right)_{(11)}
$$

From the above equation:

$$
\left\{\begin{array}{c}
\alpha_{f}=\frac{\left(l_{r} m\right)}{\left(C_{f 1} l_{f}+C_{f 1} l_{r}\right)} a_{y}-\frac{C_{f 2} l_{r}+C_{f 2} l_{f}}{\left(C_{f 1} l_{f}+C_{f 1} l_{r}\right)} \gamma_{f} \\
\alpha_{r}=\frac{\left(l_{f} m\right)}{\left(C_{r} l_{f}+C_{r} l_{r} l_{r}\right.} a_{y}-\frac{C_{r 2} l_{r}+C_{r 2} l_{f}}{\left(C_{r 1} l_{f}+C_{r 1} l_{r}\right)} \gamma_{r}
\end{array}\right.
$$

Replacing the camber angles $\left(\gamma_{f}=\phi+\delta \sin (\varepsilon), \quad \gamma_{r}=\phi\right)$ in equation (12), one gets side slip relation:

$$
\begin{aligned}
\alpha_{f}-\alpha_{r}= & \underbrace{\left(\frac{C_{r 2} C_{f 1}-C_{f 2} C_{r 1}}{C_{r 1} C_{f 1}}\right)}_{E G_{2}} \phi-\underbrace{\frac{C_{f 2}}{C_{f 1}} \sin (\varepsilon)}_{E G_{3}} \delta+ \\
= & \underbrace{\frac{\left(C_{r 1} l_{r}-C_{f 1} l_{f}\right)}{C_{f 1} C_{r 1}} \frac{m}{\left(l_{f}+l_{r}\right)}}_{E G_{1}} a_{y} \\
= & E G_{1} a_{y}+E G_{2} \phi-E G_{3} \delta
\end{aligned}
$$

From the following kinematics equations:

$$
\alpha_{f}=-\frac{v_{y}+l_{f} \dot{\psi}}{v_{x}}+\delta \cos (\varepsilon), \quad \alpha_{r}=-\frac{v_{y}-l_{r} \dot{\psi}}{v_{x}}
$$

The side slip relation is also described as:

$$
\begin{aligned}
\alpha_{f}-\alpha_{r} & =-\frac{v_{y}+l_{f} \dot{\psi}}{v_{x}}+\cos (\varepsilon) \delta+\frac{v_{y}-l_{r} \dot{\psi}}{v_{x}} \\
& =-\left(l_{f}+l_{r}\right) \frac{\dot{\psi}}{v_{x}}+\cos (\varepsilon) \delta \\
& =-\frac{\left(l_{f}+l_{r}\right)}{R}+\cos (\varepsilon) \delta
\end{aligned}
$$

The self-steering behavior depends on the sideslip difference:

$$
\left\{\begin{array}{l}
\alpha_{f}-\alpha_{r}=E G_{1} a_{y}+E G_{2} \phi-E G_{3} \delta \\
\alpha_{f}-\alpha_{r}=-\frac{\left(l_{f}+l_{r}\right)}{R}+\cos (\varepsilon) \delta
\end{array}\right.
$$

by identifying the above equations, one get:

$$
\begin{aligned}
\delta & =\frac{\left(l_{f}+l_{r}\right)}{R\left(\cos (\varepsilon)+E G_{3}\right)}+a_{y} \frac{E G_{1}}{\left(\cos (\varepsilon)+E G_{3}\right)}+\phi \frac{E G_{2}}{\left(\cos (\varepsilon)+E G_{3}\right)} \\
& =\delta_{A}+a_{y} \frac{E G_{1}}{\left(\cos (\varepsilon)+E G_{3}\right)}+\phi \frac{E G_{2}}{\left(\cos (\varepsilon)+E G_{3}\right)} \\
& =\delta_{A}+\Delta \delta
\end{aligned}
$$

With

$$
\delta_{A}=\frac{\left(l_{f}+l_{r}\right)}{R\left(\cos (\varepsilon)+E G_{3}\right)}
$$

The steering angle $\delta_{A}$ resulting from equation (17), is called the neutral steering angle. The additional $\Delta \delta$ angle is caused by the motorcycle's dynamics.

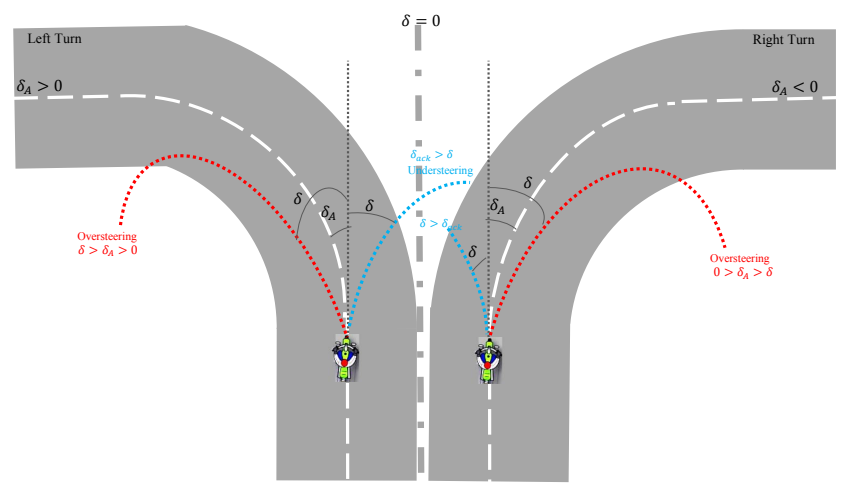

Fig. 3: Over and Under steering situation.

Now, in order to propose a detection algorithm for the over and under steer situations, we propose a risk function defined from the self steer behavior in equation (17). Therefore, the steering behavior can also be described as follows:

$$
S_{S}=\frac{\delta-\delta_{A}}{a_{y}\left(1+\frac{E G_{2} \phi}{E G_{1} a_{y}}\right)} \frac{\left(\cos (\varepsilon)+E G_{3}\right)}{E G_{1}}
$$


where $S_{S}$ represents the Self-Steer Motorcycle Gradient. In the straight-line road, the lateral acceleration is small, and $S_{S}$ values become very large. Thus we use the algebraic function $\operatorname{sign}\left(a_{y}\right)$ instead of $a_{y}$ to avoid the detection of false alarms due to $S_{S} \rightarrow \infty$. Now, the expression of the steering behavior (19) is completely defined, a simple analysis of $S_{S}$ makes it possible to characterize the steering behavior of the PTWv.

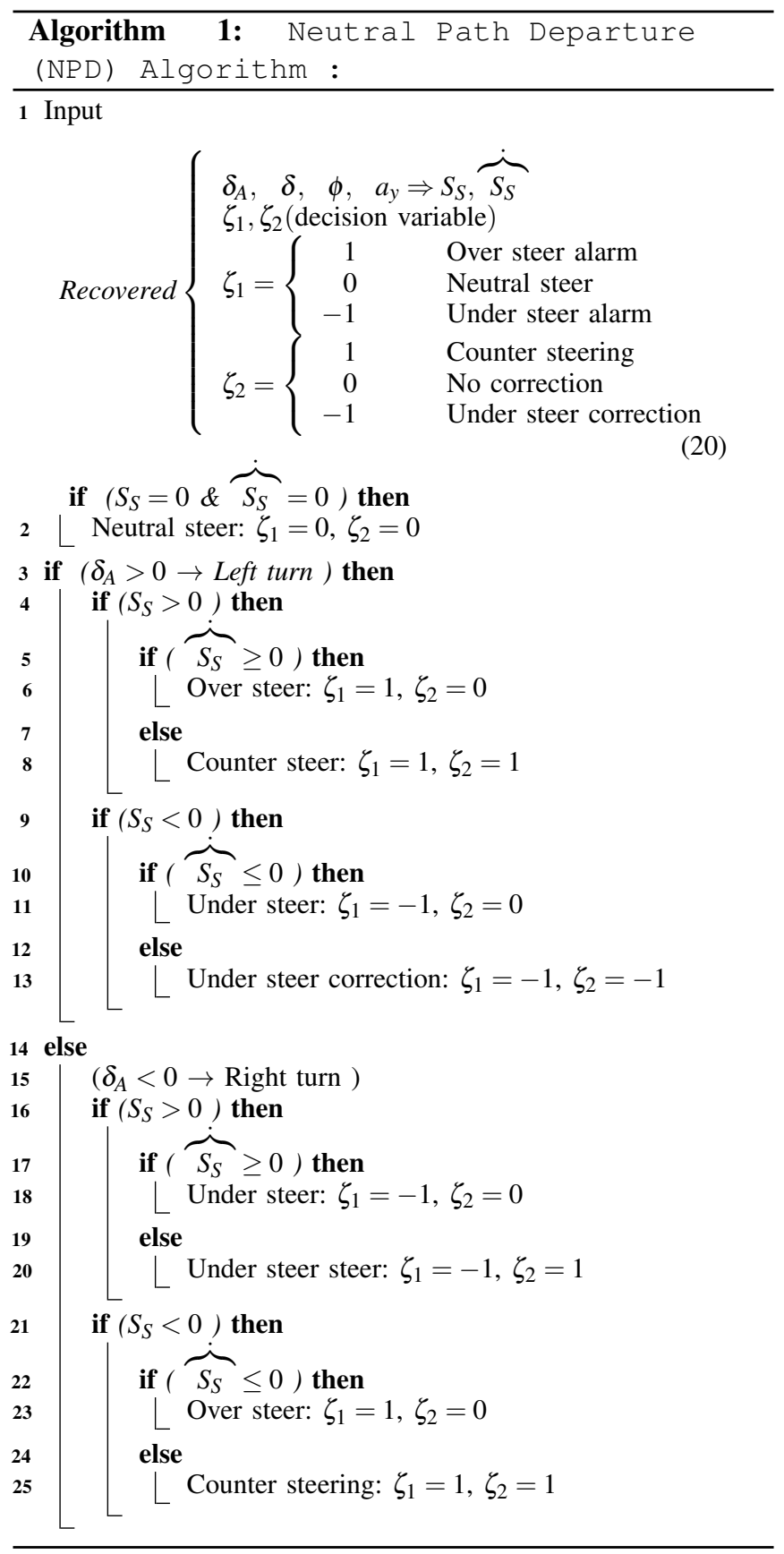

\section{Neutral Path Detection Algorithm}

Neutral Path Departure algorithm aims to help a rider in maintaining safe travel, where the goal is to detect an over or an understeer behavior compared with the neutral dynamics and to warn the rider of a lose of friction between the front and rear wheels. The NPD algorithm depends on a risk function $S_{S}$ proper for a motorcycle, this index is required to detect the drift out from the neutral steady dynamics, the sign of the $S_{S}$ signifies the understeer and oversteer behavior of the motorcycle in left and right turn. Then, the rider adjusts the steering angle, rider's posture and/or forward speed to recover the neutral trajectory without a controller. Moreover to improve the confidence of the results, the analysis of the derivative $\overbrace{S_{S}}$ is very interesting to detect the changing in the steering action if any correction is taken by the rider. This consideration is made to avoid false alarms when the driver is already correcting his maneuver. Therefore, the sign of $S_{S}$ and derivative $\overbrace{S_{S}}$ is used to define two decision variables $\left(\zeta_{1}, \zeta_{2}\right)$. The process of providing a neutral departure warning is summarized in the following algorithm (1).

The following cases are considered:

1) $S_{S} \approx \mathbf{0}(\overbrace{S_{S}}=0)$ : The motorcycle is neutral-steering $\left(\alpha_{f}=\alpha_{r}\right)$. In this case, the steering powers are equal in the front and rear wheels. When cornering, no change in steering angle is required to maintain the correct radius when the speed varies.

2) Right turn, $\delta_{A}<0$ (clockwise):

$-S_{S}<0(\overbrace{S_{S}}<0)$, when motorcycle steers towards the right: this reflects over-steering behavior. The actual cornering radius is smaller than the neutral one. Indeed, a decrease in lateral acceleration causes a greater increase in the radius of the trajectory. This phenomenon generates instability which can only be countered by a decrease in the steering angle to stay on the neutral radius.

$-S_{S}<0 \overbrace{S_{S}}^{i}>0)$ reflects counter-steering behavior (correction of the over steer)

$-S_{S}>0(\overbrace{S_{S}}^{i}>0)$ reflects under-steering behavior. It is necessary to steer the steering angle in the clockwise sense to stay on the right radius.

$-S_{S}>0 \overbrace{S_{S}}^{j}<0)$ correction of the under-steer.

3) Left turn, $\delta_{A}>0$ (anticlockwise):

$-S_{S}>0(\overbrace{S_{S}}>0)$ reflects over-steering behavior, the actual cornering radius is smaller than the neutral one, the rider has to turn the front wheel in the right side, reduce roll angle or accelerate to increase the radius and catch the neutral path.

$-S_{S}>0 \overbrace{S_{S}}^{i}<0)$ counter-steering behaviour.

$-S_{S}<0(\overbrace{S_{S}}<0)$ reflects under-steering behavior, the actual cornering radius is greater than the neutral path, the rider has to steer towards the left side or tilt to increase roll angle to reach the correct radius.

$-S_{S}<0 \overbrace{S_{S}}^{i}>0)$ under-steer correction.

Remark 1: Moreover, a hysteresis function $\operatorname{Hys}\left(S_{S}\right)$ or a memory block can be used to avoid multiple switching. This 
block holds the value of $\left(S_{S}\right)$ when the algorithm switches to test the rider correction by $\overbrace{S_{S}}$. The use of this block can minimize unwanted behavior when switching between the algorithm loops.

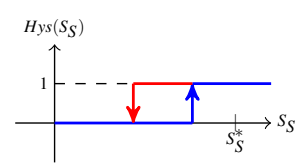

Fig. 4: Memory block

\section{Simulation}

The proposed approach is evaluated by co-simulation with Bikesim $^{\odot}$ software [24] under different riding maneuver . A PTWv model is chosen from the dataset Big Sport Baseline 8 bodies and default parameters. It will be assumed that:

- The road is flat with a high friction coefficient of $\mu=$ 0.9

- The road curvature of the neutral trajectory is considered to be constant.

- In these simulations, the motorcycle is driven at a constant speed of $50 \mathrm{~km} / \mathrm{h}$.

The simulations are carried out in two subsections:

Scenario 1: Three tests are conducted to evaluate the risk index: an oversteer scenario, a neutral turning scenario and an understeer maneuver.

Scenario 2: A mixed scenario including neutral, under, oversteer and rider correction with noise consideration to highlight the detection scheme and alarm generation.

Note that BikeSim offers several driver models with different control strategies. In our case, it is an open-loop control on the steering torque, more suitable to simulate steering behavior.

\section{A. Scenario 1}

In this subsection, it is proposed to validate the risk function selected for the detection of under and oversteer on the handlebar of a PTWv. To do this, we have simulated a circular trajectory with a constant radius of 61.4 meters for three different riding scenarios, conducted for different steering torques.

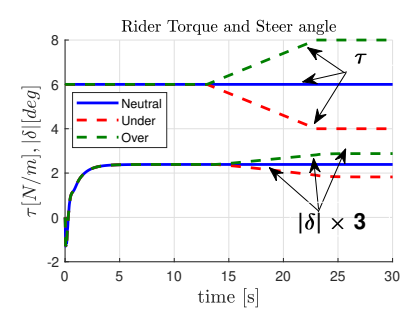

(a) Rider torque and steering angle.

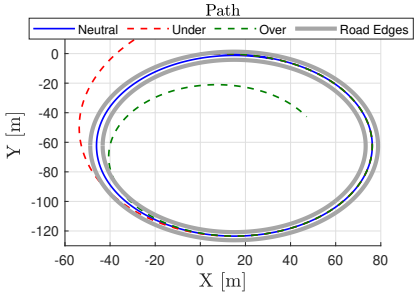

(b) Neutral Path Departure.
Fig. 5: Steering behavior.
Figure 5a shows the steering torques applied to the PTWv. On the same figure, we can also see the steering angle corresponding to a scale factor of 3 .
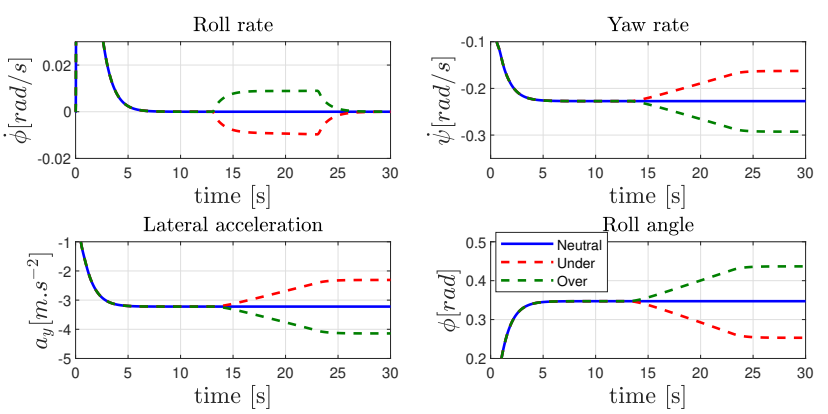

(a) Steady Outputs.
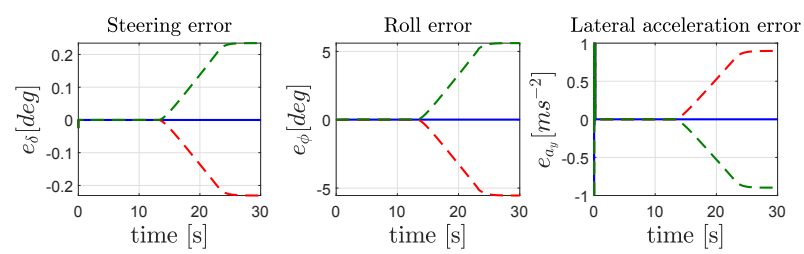

(b) State errors.

Fig. 6: Steady Steering behavior.

The input steering angle used in this tests is defined such that the wheel lift-off occurs at $13 \mathrm{sec}$, whereas in neutral test no wheel lift-off occurs. For the neutral scenario, the torque applied to the handlebar is $\tau=6 \mathrm{~N} / \mathrm{m}$. When a PTWv is oversteer, the torque applied by the rider on the handlebars is too important compared to the geometry of the turn. PTWv tends to turn inward of the curve. Conversely, when understeer the applied torque is lower than the neutral one, the PTWv tends to increase the trajectory to the road exit. The vertical dashed line refers to the time from which the steering behavior is significantly affected by the over or understeer phenomenon.

Figure $5 \mathrm{~b}$ shows the different trajectories of the PTWv during the constant turn. In blue, the motorcycle trajectory for a neutral turning. In which the motorcycle path is parallel to that of the road edges. While in red, we show the trajectories of over turning, respectively in black under turning.

Figures $6 \mathrm{a}$ and $6 \mathrm{~b}$ show the consequences of the over and under-steering phenomena on the steady state variables for the three cases. It can be seen that the slightest action on the handlebars when cornering has significant consequences on the complete dynamics of the PTWv $\left(\dot{\psi}, a_{y}, \phi\right.$, etc $)$. Figure 7 shows the steering index calculated from equation (19) for the three scenarios. It can be noted that the alarm and correction signals remain at zero when no wheel liftoff occurs. Then, these signals detect the motorcycle is drifting out: $\zeta_{1}=-1$ understeer or $\zeta_{1}=1$ oversteer. In these scenarios, no correction is taken by the ride $\zeta_{2}=0$. The $S_{S}$ shows good efficiency to early detect the steering errors from the neutral path. This advantage is very interesting since the 
neutral path departure has to be quickly avoided.
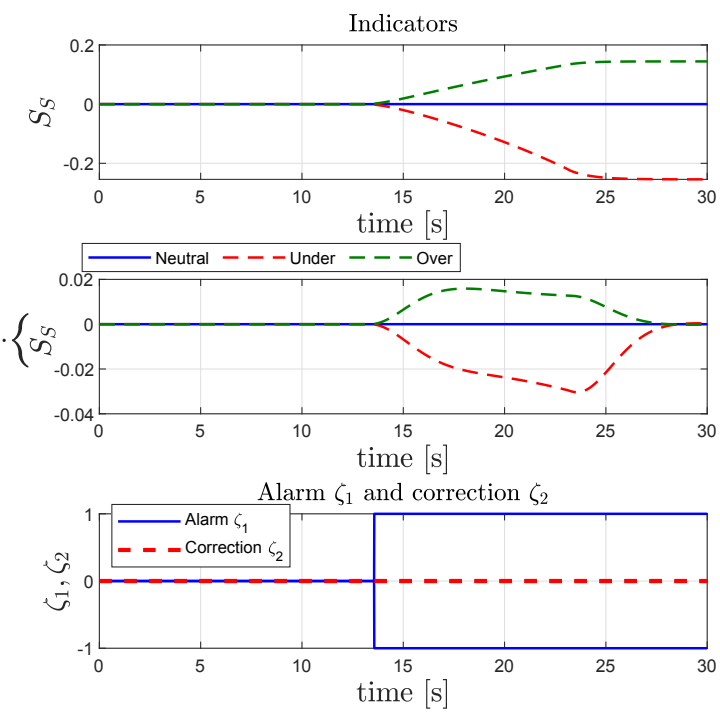

Fig. 7: Alarm and corrections.

\section{B. Scenario 2}

This part is devoted to evaluating the neutral path departure warning algorithm in noisy case.

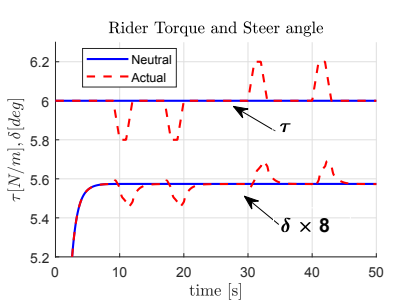

(a) Rider torque and steering angle.

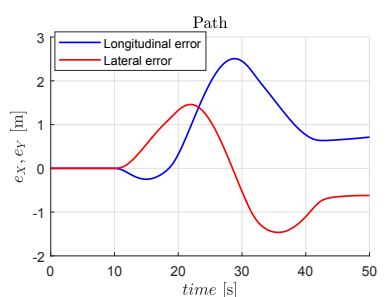

(b) Trajectory errors Departure.
Fig. 8: Steering behavior.

During this scenario, the motorcycle is driven to perfectly follow the neutral path road until $9 \mathrm{sec}$. Then, the wheel drifts out will occurs first as understeer until $30 \mathrm{sec}$ including a rider correction, then as oversteer until $50 \mathrm{~s}$ with some adjustment from the rider, seeking to catch the neutral line. Figure 8a shows the steering torques applied by the rider and the corresponding steering angle.

Fig $8 \mathrm{~b}$ shows the lateral and longitudinal errors. While, figures 9 shows the consequences of the neutral path departure on the motorcycle states.
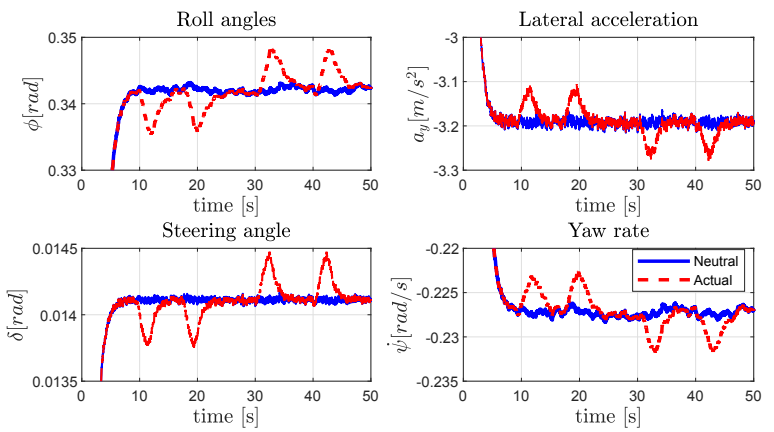

Fig. 9: Noisy outputs.

Figure 10 illustrates the relevant indicators proposed for the characterization of steering behavior. The risk indicator $S_{S}$ is computed here from the noisy measurement of the actual steering, lateral acceleration, and roll angle of the PTWv. Moreover, the analysis of $\overbrace{S_{S}}$ is very interesting to characterize the changing in the rider steering action. Note that the raw data (unfiltered) is difficult to exploit because of the noise amplified by the derivation. This is why the $S_{S}$ have been filtered with a simple first-order Butterworth filter. Therefore, we prefer to use the $S_{S}$ and derivative $\overbrace{S_{S}}$ to define two levels of risk: the first level detects the over/understeering and the second level detects if any correction is taking by rider.

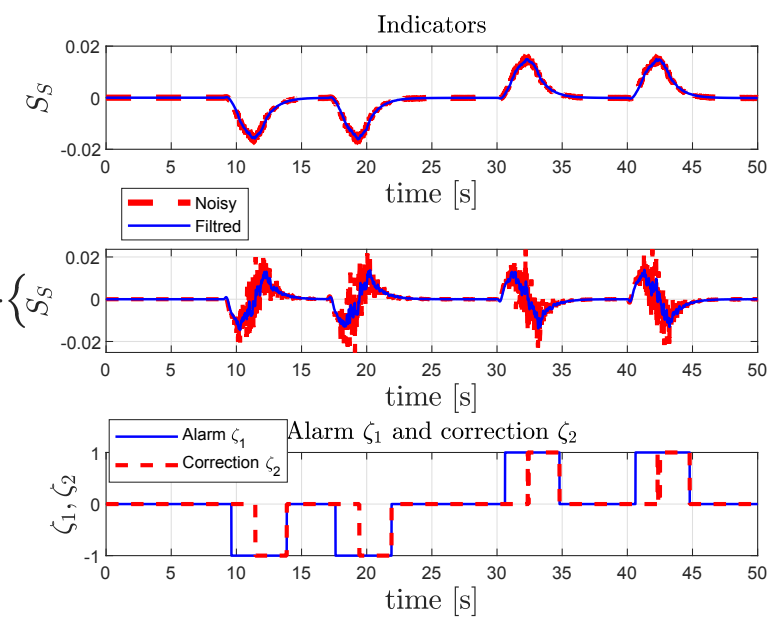

Fig. 10: Risk functions, alarm and corrections.

One can see in figures (10) that the used steering risk indicators and the alarm signal given by the detection algorithm are very interesting to detect the rider errors and the neutral path drift out even with noisy outputs. It can be noted that the alarm signal remains at zero when no neutral path departure is detected $S_{S}=0$. Then the alarm signals take the correct values depending on which direction the motorcycle is drifting out $\zeta_{1}=-1$ understeer or $\zeta_{1}=1$ oversteer. Also, one can see the alarm corresponding to rider correction $\zeta_{2}$ 
which means that the rider is trying to bring back the PTWv to the neutral path.

\section{Result discussion}

Finally, simulation results from the BikeSim software have shown that the synthesis of the detection algorithm-based risk function has undeniable potential to characterize the steering behavior. Indeed, it is much informative since it is based on the analysis of two parameters $S_{S}$ and $\overbrace{S_{S}}$. These results attest the effectiveness of the risk indicators developed for neutral path departure detection algorithm. These results highlight the effectiveness of the detection algorithm to detect in an early stage the steering deviation. This advantage is very interesting since the road departure has to be earlier avoided. Although the results are really encouraging, the idea presented in this paper deserves to be deepened. Indeed, for the validation of the approach, we are limited to the co-simulation case and improvements should be made to avoid bad detection in case of using estimated data instead of measurement outputs in some situations.

\section{CONCLUSION}

In this paper, we proposed a synthesis of a new risk function for the characterization of rider steering behavior. While conventional approaches use kinematics or geometric functions, to detect the intersection point on the road edges. We propose here a new neutral-path departure algorithm to overcome rider steering errors when the drifts out of the neutral lane. The motorcycle tendency to under or oversteer in steady turning is also analyzed, based on handling conditions. Besides, the NPD algorithm is designed based on the $S_{S}$ and $\overbrace{S_{S}}$. Then, the detection method was tested in cosimulation using BikeSim ${ }^{\odot}$ under different steering maneuvers to highlight the effectiveness of the proposed algorithm to detect in an early stage the over/under steering deviation from the neutral path, to improve motorcycle handling and correct the unsafe maneuver. Indeed, the proposed solution is very economical, limiting the amount of energy needed since it only requires a conventional IMU and a steering encoder without the need on the state observer.

\section{REFERENCES}

[1] M. B. Dominique Lord and Y. Peng, "Analysis of roadway departure crashes on two-lane rural roads in texas," in FHWA/TX-11/0-60311Washington DC, U.S. Department of Transportation, 2011.

[2] "Fatal and serious road crashes involving motorcyclists."

[3] "Safety report select risk factors associated with causes of motorcycle crashes."

[4] S. Mammar, S. Glaser, M. Netto, and J. . Blosseville, "Time-toline crossing and vehicle dynamics for lane departure avoidance," in Proceedings. The 7th International IEEE Conference on Intelligent Transportation Systems (IEEE Cat. No.04TH8749), Oct 2004, pp. 618623.

[5] S. Mammar, S. Glaser, and M. Netto, "Time to line crossing for lane departure avoidance: a theoretical study and an experimental setting," IEEE Transactions on Intelligent Transportation Systems, vol. 7, no. 2, pp. 226-241, June 2006

[6] W. Wang, D. Zhao, W. Han, and J. Xi, "A learning-based approach for lane departure warning systems with a personalized driver model," IEEE Transactions on Vehicular Technology, vol. 67, no. 10, pp. 91459157, Oct 2018.
[7] A. Benine-Neto, S. Scalzi, S. Mammar, M. Netto, and B. Lusetti, "Model reference-based vehicle lateral control for lane departure avoidance," International Journal of Vehicle Autonomous Systems, vol. 12, no. 3, pp. 284-306, 2014.

[8] S. Lefevre, Y. Gao, D. Vasquez, H. E. Tseng, R. Bajcsy, and F. Borrelli, "Lane keeping assistance with learning-based driver model and model predictive control," in 12th International Symposium on Advanced Vehicle Control, 2013.

[9] Y. Marumo and N. Katagiri, "Control effects of steer-by-wire system for motorcycles on lane-keeping performance," Vehicle System Dynamics, vol. 49, no. 8, pp. 1283-1298, 2011. [Online]. Available: https://doi.org/10.1080/00423114.2010.515030

[10] N. Katagiri, Y. Marumo, and H. Tsunashima, "Evaluating lanekeeping-assistance system for motorcycles by using rider-control model," in SAE Technical Paper. SAE International, 092008. [Online]. Available: https://doi.org/10.4271/2008-32-0056

[11] _ , "Controller design and evaluation of lane-keeping-assistance system for motorcycles," Journal of mechanical systems for transportation and logistics, vol. 2, no. 1, pp. 43-54, 2009.

[12] E. Y. Chung, H. C. Jung, E. Chang, and I. S. Lee, "Vision based for lane change decision aid system," in 2006 International Forum on Strategic Technology, Oct 2006, pp. 10-13.

[13] P.-M. Damon, H. Hicham, H. Arioui, and K. Youcef-Toumi, "Powered two-wheeled vehicles steering behavior study: Vision-based approach," 11 2018, pp. 355-360.

[14] P. Damon, H. Hadj-Abdelkader, H. Arioui, and K. Youcef-Toumi, "Image-based lateral position, steering behavior estimation, and road curvature prediction for motorcycles," IEEE Robotics and Automation Letters, vol. 3, no. 3, pp. 2694-2701, July 2018.

[15] H. Dahmani, M. Chadli, A. Rabhi, and A. El Hajjaji, "Fuzzy uncertain observer with unknown inputs for lane departure detection," in Proceedings of the 2010 American Control Conference. IEEE, 2010, pp. 688-693.

[16] H. B. Pacejka, "Simplified analysis of steady-state turning behaviour of motor vehicles. part 1. handling diagrams of simple systems." Vehicle System Dynamics, vol. 2, no. 3, pp. 161-172, 1973.

[17] E. Velenis, E. Frazzoli, and P. Tsiotras, "On steady-state cornering equilibria for wheeled vehicles with drift," in Proceedings of the $48 \mathrm{~h}$ IEEE Conference on Decision and Control (CDC) held jointly with 2009 28th Chinese Control Conference. IEEE, 2009, pp. 3545-3550.

[18] V. V. Grigorievich, B. A. Igorevich, and T. D. Nikolayevich, "Handling and stability analysis of vehicle plane motion," Mathematics and Computer Science, vol. 3, no. 1, p. 13, 2018.

[19] U. Wasiwitono, I. N. Sutantra, Y. Triwinarno et al., "Steady-state cornering modeling and analysis of three-wheel narrow vehicle," in Applied Mechanics and Materials, vol. 758. Trans Tech Publ, 2015, pp. 173-178.

[20] S. Evangelou, "Control and stability analysis of two-wheeled road vehicles," Ph.D. dissertation, University of London London, 2004.

[21] S. Glaser, S. Mammar, and C. Sentouh, "Integrated driver-vehicleinfrastructure road departure warning unit," IEEE Transactions on Vehicular Technology, vol. 59, no. 6, pp. 2757-2771, 2010.

[22] E. Velenis, E. Frazzoli, and P. Tsiotras, "Steady-state cornering equilibria and stabilisation for a vehicle during extreme operating conditions," International Journal of Vehicle Autonomous Systems, vol. 8, no. 2-4, pp. 217-241, 2010.

[23] V. Cossalter, Motorcycle dynamics. Lulu. com, 2006.

[24] R. Sharp, S. Evangelou, and D. J. Limebeer, "Advances in the modelling of motorcycle dynamics," Multibody system dynamics, vol. 12, no. 3, pp. 251-283, 2004. 